\title{
Psicologia e Educação a Distância: Uma Revisão Bibliográfica
}

\author{
Psychology And Distance Education: \\ A Literature Review \\ Psicología Y Educación A Distancia: \\ Una Revisión De La Literatura
}
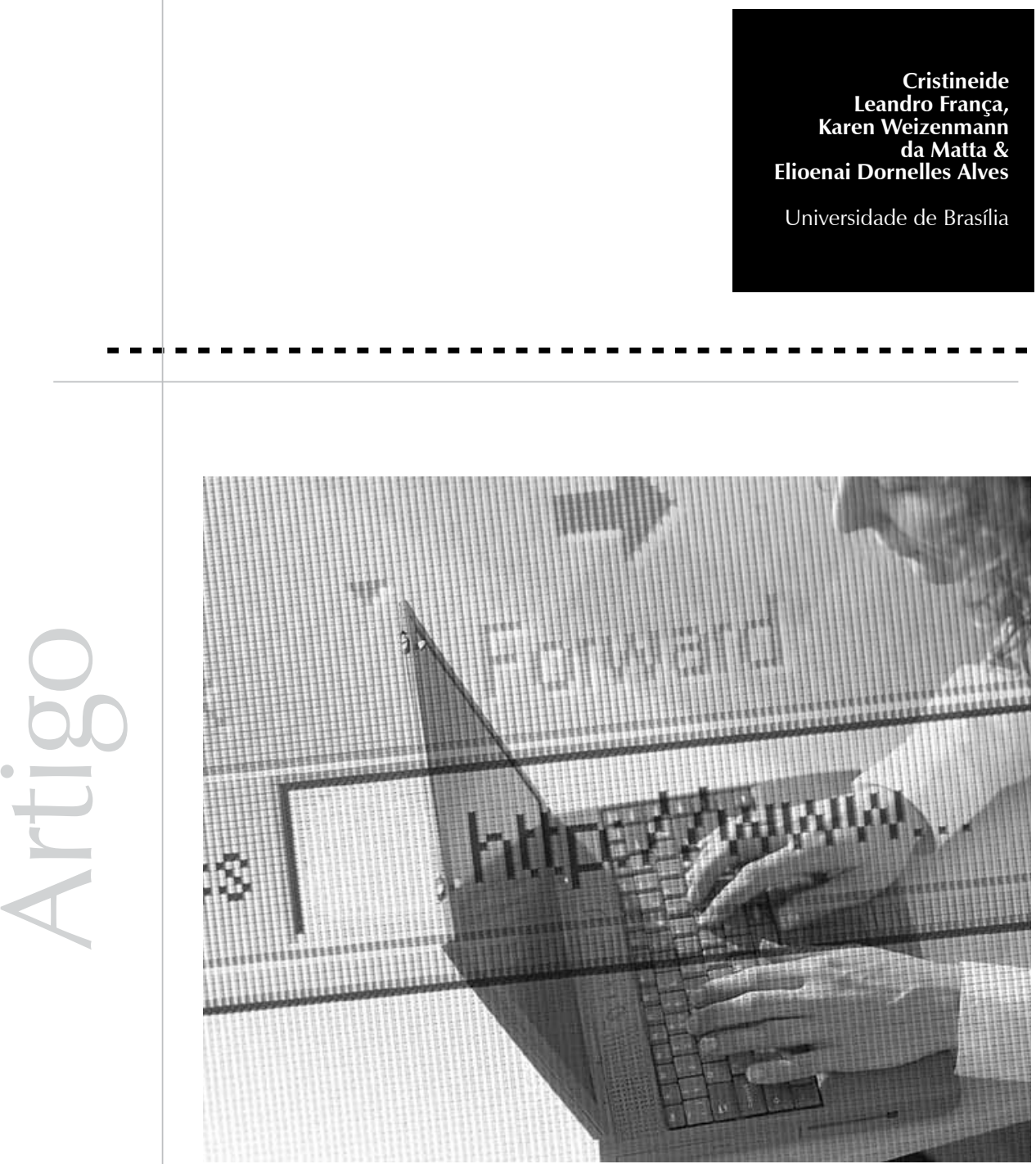
Resumo: O presente estudo investigou as contribuições da Psicologia para a educação a distância - EAD. Para isso, realizou-se revisão bibliográfica de publicações nacionais, entre 1999 e 2009, nas bases de dados SciELO, Lilacs, PsycINFO, BVS e Google Acadêmico. Foram selecionados 69 estudos, dos quais se excluíram 15, por não serem compatíveis com a pesquisa. Os resultados evidenciaram nove eixos temáticos: (1) tecnologia educacional a distância com fundamentos psicológicos, (2) afeição, (3) papel do tutor/professor, (4) teorias psicológicas, (5) interatividade, (6) evasão, (7) relatos de experiências, (8) avaliação de treinamento, desenvolvimento e educação a distância e (9) outros. Os temas tecnologia educacional a distância com fundamentos psicológicos e afeição obtiveram maior frequência nas publicações. Neste estudo, registrou-se, também, que, embora em ascensão desde 2006, os anos de maiores publicações da Psicologia na educação a distância foram 2008 e 2009. Sugere-se que estudos futuros ampliem esta pesquisa para incluir bases de dados internacionais.

Palavras-chave: Psicologia. Educação a distância. Tecnologia educacional. Afeição.

Abstract: This study investigated the contributions of Psychology to Distance Learning. A literature review of national publications between 1999 to 2009 was made in the electronic databases SciELO, LILACS, PsycINFO, BVS and Google Scholar. From the 69 studies selected, 15 were excluded because they were not compatible with the research. The results showed nine themes: (1) the distance learning technology with psychological grounding, (2) affection, (3)the role of the tutor/professor, (4) psychological theories, (5) interactivity, (6) dropout, (7) reports of experiences, (8) assessment of distance training, development and education and (9) others. The topics distance education technology with psychological grounding and affection had a larger frequency in the publications. Since 2006, there was an increasing number of publications in psychology distance education, but the years of major publications were from 2008 to 2009. It is suggested that future studies expand this research including international databases.

Keywords: Psychology. Distance education. Educational technology. Affection.

Resumen: El presente estudio investigó las contribuciones de la Psicología para la Educación a Distancia EAD. Para eso, fue realizada una revisión bibliográfica de publicaciones nacionales, entre 1999 y 2009 en las bases de datos SciELO, Lilacs, PsycINFO, BVS y Google Académico. Fueron seleccionados 69 estudios, de los cuales se excluyeron 15, por no ser compatibles con la pesquisa. Los resultados evidenciaron nueve ejes temáticos (1) tecnología educacional la distancia con fundamentos psicológicos; (2) afección; (3) papel del tutor/profesor; (4) teorías psicológicas; (5) interactividad; (6) evasión; (7) relatos de experiencias, (8) evaluación de entrenamiento, desarrollo y educación distancia y (9) otros. Los temas tecnología educacional a distancia con fundamentos psicológicos y afección obtuvieron mayor frecuencia en las publicaciones. En ese estudio se registró, también, que, aunque en ascensión desde 2006, los años de mayores publicaciones de la psicología en la educación a distancia, fueron 2008 y 2009. Se sugiere que estudios futuros amplíen esa pesquisa para incluir bases de datos internacionales.

Palabras clave: Psicología. Educación a distancia. Tecnología educacional. Afección.

A educação a distância - EAD surge para estimular e disseminar o conhecimento ao construir uma aprendizagem colaborativa e romper características da educação tradicional, entre elas tempo e rigidez física (Formiga, 2009). Entretanto, o processo de aprendizagem em EAD vai além das questões espaço/tempo, pois envolve peculiaridades psicossociais que dificultam ou contribuem para o conhecimento ao englobar as questões afetivas e interativas desse processo. A Psicologia se destaca entre as ciências que contribuem para o desenvolvimento da EAD ao estudar principalmente as considerações psicológicas que influenciam as relações professor/aluno, o fenômeno da interatividade nessa abordagem tecnológica e as teorias educacionais que a fundamentam. Ao abordar as contribuições da Psicologia para a educação a distância, é necessária uma conceituação da EAD em uma perspectiva histórica bem como a análise de como essas ciências se relacionam.

\section{Educação a distância: definições e história}
A EAD pode ser definida como uma modalidade educacional que ocorre principalmente com professores e alunos fisicamente separados todo o tempo ou grande parte do tempo, mas que se comunicam por 
Trata-se de um sistema tecnológico, um modelo industrializado da educação (Peters,

1994), com independência intelectual (Moore, 1972), que utiliza recursos como textos impressos, eletrônicos e mecânicos para facilitar a comunicação e propiciar aprendizagem aos alunos de forma flexível e autônoma. meio das tecnologias de informação e de comunicação. Estudiosos clássicos nesse tema (Moore, 1972; Aretio, 1994; Peters, 1994) apontam a EAD como um método de instrução autônomo, de troca e comunicação bidirecional, que apresenta características e comportamentos diferenciados entre professor e aluno. Trata-se de um sistema tecnológico, um modelo industrializado da educação (Peters, 1994), com independência intelectual (Moore, 1972), que utiliza recursos como textos impressos, eletrônicos e mecânicos para facilitar a comunicação e propiciar aprendizagem aos alunos de forma flexível e autônoma.

$\mathrm{Na}$ história mundial, o fato que marcou formalmente o surgimento da EAD ocorreu em 1881, quando William R. Harper, fundador e primeiro reitor da Universidade de Chicago, ofereceu um curso de hebreu por correspondência (Santana, Gaspar, Costa, Paiva, Rodrigues, \& Alves, 2005). Mas, de acordo com a literatura (Nunes, 2009), em meados de 1728, esse novo método de ensinar a distância já era realizado por Caleb Philips, que enviava lições aos seus alunos para serem publicadas na Gazette de Boston, EUA. Nas décadas seguintes, mais especificamente nos anos 1840, 1880 e 1884, foram oferecidos na Grã-Bretanha, respectivamente, cursos de taquigrafia por correspondência, preparatórios para concursos públicos e cursos de contabilidade, e, em 1991, nos Estados Unidos, curso sobre segurança de minas. Entretanto, a verdadeira ascensão da EAD ocorreu em meados da década de 60 do século XX, iniciando pela Europa e passando depois aos demais continentes, com ações dirigidas à educação secundária e superior (Perry \& Rumble, 1987). Atualmente, no cenário mundial, mais de 80 países atendem a milhões de estudantes por meio da EAD.

A supracitada história mundial da EAD é classificada em distintas gerações, de acordo com o desenvolvimento dos meiostecnológicos. A primeira geração foi caracterizada pelo ensino por correspondência e a segunda, pela geração pelos telecursos $\mathrm{O}$ paradigma predominante das duas primeiras gerações foi a EAD como modelo industrializado de educação, com base em princípios de racionalização, produção de massa, mecanização e automação (Romiszowski, 2009). A terceira geração é caracterizada pelo modelo tecnológico interativo, como a internet e a videoconferência. No começo do século XXI, surgem discussões sobre o nascimento da quarta e da quinta gerações marcadas pelo uso da inteligência artificial e de realidade virtual (Torres \& Fialho, 2009). Os paradigmas predominantes dessas novas gerações consistem em aprendizagem colaborativa e filosofias construtivistas, caracterizadas por autonomia, flexibilização e individuação.

No contexto brasileiro, a EAD teve origem há mais de um século. Essa trajetória teve início no Rio de Janeiro, pouco antes de 1900, por meio de anúncios em jornais de circulação, que ofereciam cursos de datilografia por correspondência. Nas décadas seguintes, com o advento do rádio, da televisão, dos computadores, da internet, da criação de universidade aberta e das legislações aplicáveis à $E A D$, essa modalidade de ensino tornou-se abrangente e promissora. Na visão de Alves (2009), o contexto histórico da EAD no Brasil dividiu-se em três fases: inicial, intermediária e moderna. As etapas inicial e intermediária foram marcadas positivamente, em 1904, pela instalação das escolas internacionais que ofereciam cursos para as pessoas que buscavam empregos, em 1923, pela criação dos programas populares educativos da Rádio Sociedade do Rio de Janeiro e, em 1973, no âmbito da educação superior, pela criação de uma base para programas de projeção na Universidade de Brasília. No período mais moderno, registra-se a influência de três organizações que contribuíram de maneira 
significativa para o desenvolvimento da EAD: a Associação Brasileira de Teleducação - ABT (1971), o Instituto de Pesquisas Avançadas em Educação - Ipae (1973) e a Associação Brasileira de Educação a Distância - ABED (1995). Essas organizações são responsáveis pela realização de seminários, encontros, congressos, produções científicas, edições de revistas, implantação de cursos, formulação de políticas e disposições normativas, criação de secretaria no Ministério da Educação e articulação entre instituições e profissionais no País e no exterior. No ranking internacional, o Brasil configurou-se como um dos principais países no desenvolvimento da EAD, principalmente até os anos 70 . Após essa década, houve um período de estagnação, com novo crescimento apenas no final do milênio (Alves, 2009).

O cenário atual do ensino a distância assume uma natureza interdisciplinar (Litto, 2009) marcado pela troca de conhecimentos científicos. Nesse campo, as inovações tecnológicas se mostram rápidas e contínuas, consubstanciadas pela inclusão de universidades corporativas, universidades abertas a distância, inclusão digital, novos modelos pedagógicos, como a heutagogia (aprendizagem autodeterminada), redes educacionais abertas, o advento da Web, de televisão digital, do moodle e do móbile learning. Destaca-se, entre as inovações, o amplo uso de diversos métodos de ensino e da aprendizagem on-line, o que se denominou e-learning. As pesquisas sobre e-learning estão relacionadas aos aspectos pedagógicos, à interação homem/computador, à estratégia de e-learning, aos aspectos tecnológicos, à afirmação e ao controle de qualidade, à gestão de sistemas e de recursos, aos aspectos culturais e ao desenvolvimento de habilidades e competências por meio de e-learning. Há necessidade de estudos futuros sobre os seguintes tópicos: uso de tecnologias móveis/ celulares para EAD, montagem e uso eficaz de comunidades de prática on-line, inovações na área de design instrucional, novas formas de controle e avaliação dos resultados da aprendizagem on-line, adaptação dos ambientes de aprendizagem on-line às características individuais dos estudantes e modelos de ensino-aprendizagem adequados à EAD (Romiszowski, 2009).

\section{Educação a distância e Psicologia}

O papel da Psicologia no ensino a distância fundamenta-se principalmente em contribuições encontradas nos modelos de teorias do desenvolvimento, da aprendizagem, cognitiva, comportamental e socioconstrutivista. Destacam-se, nesse campo, os estudos sistemáticos sobre aprendizagem de Pavlov, Watson e Thorndike decorrentes da conexão entre estímulos e resposta, de Skinner, com as modificações do comportamento, e, principalmente, a descoberta do método de instrução programada, Piaget e as concepções genético-evolutivas, nas áreas cognitivas e do desenvolvimento humano, e Vygotsky, com o conceito de zona de desenvolvimento proximal (Filatro, 2009; Bizzo, 2009). É crescente o interesse dos psicólogos em investigar a relação entre fatores psicológicos e tecnologia da informação. A interação homem-computador, o relacionamento pela internet, a interação professor-aluno, a computação afetiva e o ambiente virtual, entre outros, são temas frequentes em estudos da área. Ao revisar a produção científica brasileira em EAD, Santos, Neto J., Araújo, Oliveira, Barbosa e Zander (2007) verificaram que a Psicologia está tendo maior inserção, com foco na análise do comportamento das pessoas envolvidas e atuantes em EAD. Entretanto, ainda são escassos os estudos que analisam a influência da Psicologia na evolução da educação a distância.

Para a Associação Brasileira de Ensino de Psicologia (ABEP, 2007), a participação da Psicologia nos estudos sobre EAD faz-se premente, haja vista a enorme expansão 
da EAD em decorrência do incentivo do Governo Federal. As perspectivas (reais ou especulativas) de se aumentar o acesso à educação superior com baixo custo possibilitaram a reflexão sobre o credenciamento de cursos de Psicologia a distância no País. Diante disso, em março de 2007, a ABEP criou oficialmente um Grupo de Trabalho (GT) sobre EAD para discutir a relação dessa modalidade de educação com a Psicologia (ABEP, 2007). Considerando a relevância da questão, o presente estudo buscou responder quais são as contribuições da Psicologia para a EAD. O objetivo geral consiste em analisar as contribuições da Psicologia para a EAD no período de 1999 a 2009, e os específicos são: (1) identificar as contribuições da Psicologia para a EAD e (2) classificá-las em eixos temáticos.

\section{Método}

Foi realizada uma revisão de literatura das publicações nacionais sobre EAD e Psicologia, entre 1999 a 2009, no SciELO, Lilacs, PsycINFO, BVS e Google Acadêmico. O método consistiu em sintetizar as principais contribuições da Psicologia para a educação a distância em eixos temáticos por meio da análise de conteúdo (Bardin, 2009). Embora alguns estudos abordem no mesmo trabalho a interlocução entre várias temáticas, buscou-se classificar os eixos temáticos conforme o objetivo principal e de maior relevância psicológica para a EAD. Os estudos selecionados incluíram dissertações, teses, artigos científicos e comunicações em congressos. Foram excluídos os textos com enfoque comercial ou de divulgação e os que não apresentavam relação entre a Psicologia e EAD como objeto central da reflexão. Foi realizada uma busca pelas associações das seguintes palavras-chave: Psicologia e educação a distância, estudos da Psicologia em EAD, fatores psicológicos e EAD. Os textos selecionados foram organizados, inicialmente, conforme o ano de publicação, o(os) autor(es), o título e o tipo de publicação. Em seguida, as contribuições da Psicologia para a EAD foram identificadas e classificadas em eixos temáticos, a partir da leitura das publicações.

\section{Resultados e discussão}

Foram identificados 69 estudos, a partir do ano 1999. Após a análise dos títulos e de seus respectivos resumos, de acordo com os critérios de inclusão e de exclusão, excluíramse 15 textos e utilizaram-se 54 para análise. Excluíram-se 14 textos que não tinham como objetos centrais de investigação a Psicologia e a educação a distância e 01 em que não constava o ano de publicação. A partir da leitura dessas publicações selecionadas, nove eixos temáticos foram construídos: (1) tecnologias educacionais com fundamentos psicológicos, (2) afeição, (3) papel do tutor/ professor, (4) teorias psicológicas, (5) interatividade, (6) evasão, (7) relatos de experiências, (8) avaliação de treinamento, desenvolvimento e educação a distância e (9) outros.

Houve maior número de artigos no eixo temático tecnologias educacionais com fundamentos psicológicos (vide Tabela 1). Essa categoria compreende ferramentas tecnológicas que utilizam conhecimentos psicológicos para facilitar a aprendizagem, como, por exemplo, a computação afetiva, as tecnologias interativas e a utilização de estratégias computacionais. Destaca-se, nessa categoria, a computação afetiva, uma área recente (1990) que traz benefícios à EAD ao relacionar objetos de estudo da Psicologia como processos cognitivos, afetivos e emocionais à interação homem-computador por meio de softwares e hardwares que facilitam o processo ensino-aprendizagem.

No eixo denominado afeição, que obteve o segundo maior número de publicações, os estudos citaram a interferência desses fatores na aprendizagem e o uso de ferramentas tecnológicas como fórum de discussão, mural e bate papo como formas de expressar opiniões, experiências e emoções. Os dados confirmam estudos que enunciam 
que emoções, sentimentos, experiências sensíveis, medo, raiva e interações empáticas fazem parte das inter-relações no processo de educação a distância (Lopes \& Xavier, 2007; Bercht, 2006). Assim, um dos grandes desafios a ser enfrentado pelas instituições provedoras de educação a distância referese mais a questões de ordem socioafetiva do que propriamente a conteúdos ou métodos. Nesse sentido, alguns modelos computacionais definidos como afetivos têm auxiliado no processo de ensinoaprendizagem em EAD.

Nesse segmento, observa-se que as categorias papel do tutor/professor e as teorias psicológicas aplicadas à EAD tiveram a mesma frequência de estudos selecionados. O papel do tutor/ professor refere-se às competências do docente virtual, ou seja, seus conhecimentos, habilidades, atitudes e formas de desenvolver e potencializar as capacidades básicas dos alunos. Para tanto, esse papel requer, além de competências técnicas, didáticas e metodológicas (Andrade, 2007), habilidades de mediação e de sensibilidade do educador para perceber e auxiliar nas dificuldades inerentes ao ambiente virtual como linguagem e interação.

As teorias psicológicas englobam um conjunto de arcabouços teóricos da Psicologia utilizados em EAD, tais como as teorias de Piaget e Vygotsky, a psicanálise, a análise psicodramática e os tipos psicológicos. A teoria construtivista de Piaget e a sociointeracionista de Vygotsky despertam grande interesse dos pesquisadores da área de ensino a distância, Psicologia e computação. O processo de adaptação e equilibração, por meio da assimilação e acomodação de Piaget, e o conceito de zona de desenvolvimento proximal, de Vygotsky, podem ser aplicáveis aos ambientes virtuais, desde que adaptados com a continuação de encontros presenciais, mesmo que esporádicos, e com interatividade contínua (Pereira, Pavanati, Maia, Fialho, Paulista, Schiuber, 2007). Com base na psicanálise, foram estudados aspectos que abordam as questões presença-distância e o de centralização no aluno (Voltolini, 2009). As teorias do psicodrama propõem reflexões sobre a natureza psicossocial na educação a distância, incluindo características como: separação professor-aluno, aprendizagem flexível e comunicação bidirecional (Sá, 2007). A utilização do modelo de personalidade baseada em tipos psicológicos definidos por Cal Gustav Jung contribuiu para a criação de um protótipo de curso a distância com o objetivo de avaliar a melhoria da aprendizagem e auxiliar na identificação do perfil de cada aluno (Passarela, 2007).

A próxima categoria, em ordem decrescente de frequência de publicação, é a interatividade, que envolve estudos sobre as relações, as mediações e as comunicações entre tutor, aluno e material. Moore (como citado em Mattar, 2009) foi um dos precursores nos estudos sobre interatividade em EAD ao abordar três tipos de interação: aluno/ professor, com destaque para o feedback do tutor, sem demora, como forma de auxiliar no aprendizado; aluno/conteúdo, com ênfase no uso das tecnologias modernas como internet, som, vídeo, texto e imagens para facilitar a assimilação do conteúdo, e, por fim, a interação aluno/aluno, caracterizada como aprendizado colaborativo e cooperativo entre os colegas, que desenvolve a motivação, o senso crítico, a sensação de pertencimento e diminui o isolamento.

Em seguida, observam-se as categorias evasão e relatos de experiências, que abordam, respectivamente, o fenômeno de desistência ou de abandono em EAD e as descrições de práticas em EAD. A categoria avaliação em treinamento, desenvolvimento e educação TD\&E a distância inclui a análise da reação, do aprendizado, do comportamento e dos resultados dos atores envolvidos em EAD. Estudos sistemáticos da Psicologia instrucional e organizacional sobre a eficácia das ações educacionais à distância oferecidos em ambientes corporativos e profissionais buscam identificar maneiras de garantir o 
desempenho no trabalho, a aquisição de conhecimentos, as habilidades e as atitudes por meio de estratégias de TD\&E (Zerbini, 2007). Por fim, os temas com baixa frequência para constituir um eixo temático distinto, tais como representações sociais, tensões, análise dos processos cognitivos, fatores socioafetivos, estilos de aprender e atitudes em EAD foram reunidos no eixo temático outros.

Tabela 1. Publicações sobre EAD e Psicologia, de 1999 a 2009, classificadas em eixos temáticos, número de publicações, autoria e ano de publicação

\begin{tabular}{lll}
\hline $\begin{array}{l}\text { Eixos temáticos } \\
\text { em EAD }\end{array}$ & $\begin{array}{l}\text { Número de } \\
\text { publicações }\end{array}$ & Autoria e ano \\
\hline $\begin{array}{l}\text { Tecnologias edu- } \\
\text { cacionais com }\end{array}$ & 10 & Todorov, Moreira, \& Martone (2009); Longhi,, Pereira Berchet, \\
fundamentos & & \& Behar (2009); Jaques, Lehmann, \& Jaques (2008); Longhi, \\
psicológicos & & Berchet, \& Behar (2008); Behar, Bercht, \& Longhi (2007); \\
& Tamariz, González, Pessanha, \& Braga (2007); Jaques \& Vicari \\
& (2005); Bercht (2006); Martins, Melo, Meireles, \& Nalini (2003); \\
& Simão (2001).
\end{tabular}

Afeição

Papel do tutor/ 7 professor

Teorias psicológicas aplicadas à

EAD

Interatividade

6

Evasão

Relatos de ex-

periências

Avaliação em

treinamento, desenvolvimento e educação a distância
Tijiboy, Pereira, \& Woiciechoski (2009); Santos (2009); Cunha, Silva, \& Berchet (2008); Weber (2008); Silva \& Heck (2008); Lopes \& Xavier, (2007); Serra (2005); Fiuza (2002).

Tijiboy, Carneiro, Woiciechoski, \& Pereira (2009); Gouvêa \& Cesar, (2009); Figueiredo \& Araújo (2009); Guilherme \& Monteiro (2009); Ziviani, Melli, Neto, Araújo, \& Santos (2008); Andrade (2007); Sarmet \& Abrahão, (2007).

Voltolini (2009); Azambuja \& Guareschi (2007); Passarela (2007); Pereira et al. (2007); Sá (2007); Machado \& Miranda, (2006); Oliveira, Capelo, Rego, \& Villardi (2004).

Vedove \& Camargo (2009); Laguardia, Machado, \& Coutinho (2009); Bassini, Lahud, \& Lima (2009); Amaral \& Rosini (2009); Lawisncky, Haguenauer, Cordeiro, \& Vinicius (2008); Mehleche, Pereira, \& Tarouco (2005).

Jensen \& Almeida (2009); Pinto (2009); Ramminger (2006); Abbad, Carvalho, \& Zerbini (2006).

Sarmento, Barbosa, \& Mangan (2008); Borba, Range, \& Elia (2007); Rangé (2001); Azevedo, Guerra, Zweiman. \& Nishizawa (2001).

Zerbini \& Abbad (2009); Zerbini (2007); Carvalho \& Abbad (2006).
Outros

Valentini \& Bisol, (2008); Santos (2006); Quarto (2006); Saraiva, Pernigott, Barcia. \& Lapolli (2006); Steil, Pillon, \& Kern (2005). 
No que se refere ao número de estudos publicados por ano, conforme as bases pesquisadas, a Figura 2 mostra que, entre 1999 e 2000, nenhum artigo foi encontrado; os primeiros artigos localizados são de 2001. De 2002 a 2004, houve uma redução do número de publicações, apenas 01 por ano. Em 2005, ocorreu uma retomada do crescimento, e, a partir de 2006, os números de publicações subiram de forma significativa. Observa-se que 2009 foi o ano com mais publicações que relacionam Psicologia e EAD.

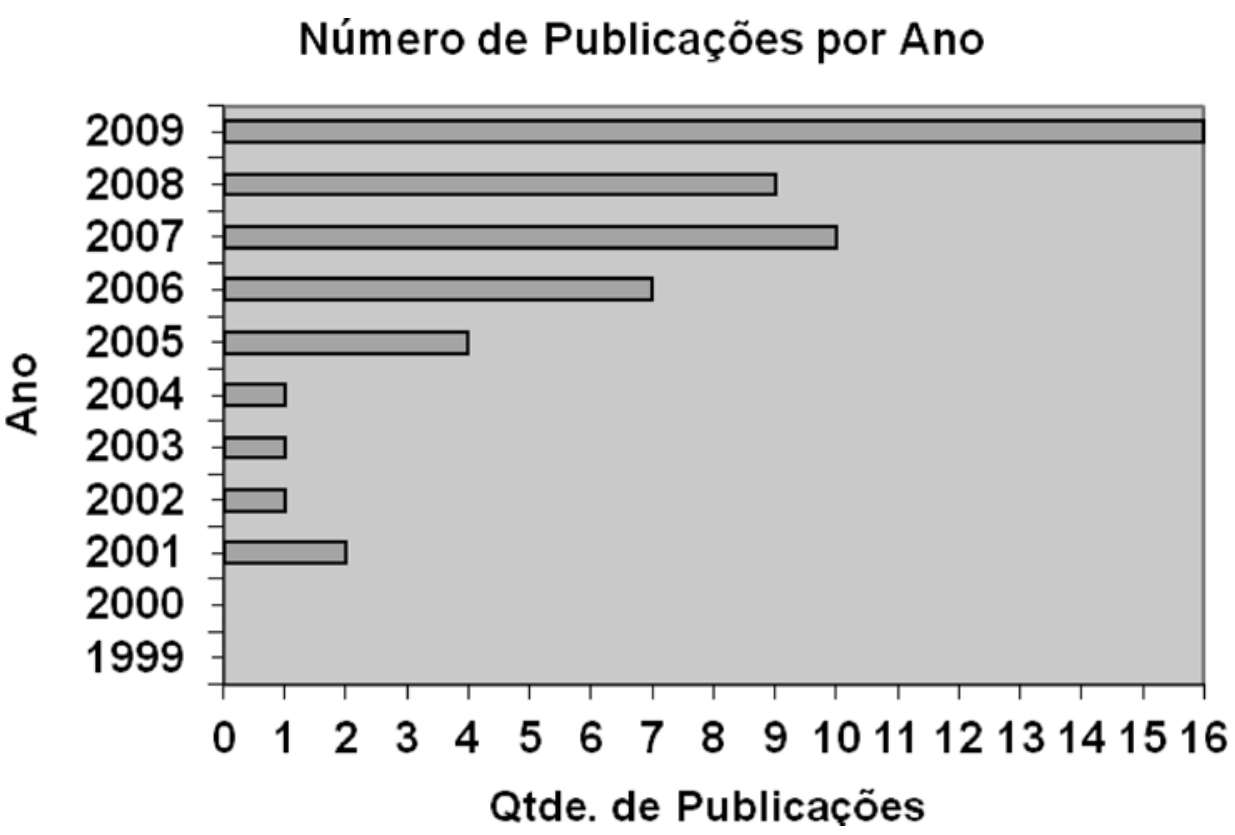

Figura 2. Número de publicações, por ano, que relacionam Psicologia e EAD entre 1999 e 2009

\section{Considerações finais}

O presente estudo conseguiu identificar algumas contribuições da Psicologia para a EAD entre 1999 e 2009. A revisão bibliográfica das bases SciELO, Lilacs, PsycINFO, Biblioteca Virtual de Saúde-BVS e Google Acadêmico demonstrou que o papel da Psicologia junto à EAD encontra relevância não apenas nas contribuições teóricas psicológicas acerca da interação professor-aluno, alunos-colegas e questões afetivas mas também na adequação das ferramentas tecnológicas às subjetividades de cada aluno.

Os resultados encontrados apontam um crescente interesse pela temática Psicologia e EAD nos últimos 10 anos, com a predominância de um número expressivo de textos que abordam as tecnologias educacionais com fundamentos psicológicos, principalmente aqueles relacionados aos estudos sobre a computação afetiva. Os dados permitem concluir que houve predominância de publicações com os temas relacionados a Psicologia e EAD nos quatro últimos anos, principalmente nos anos 2008 e 2009. Esses dados são coerentes com os estudos de Santos et al. (2007), que, ao revisar a produção científica brasileira em EAD, aponta que, a partir de 2006, houve uma inserção dos temas da Psicologia, a qual tem buscado analisar o comportamento das pessoas 
envolvidas e atuantes em EAD. Nesse sentido, é possível afirmar, assim como Santos et al. (2007), que o aumento de pesquisas em Psicologia em EAD é um aspecto interessante, no qual diferentes visões são lançadas acerca do comportamento das pessoas, de forma a propiciar feedback aos planejadores com vistas a fortalecer a qualidade em EAD. Entretanto, estudos sobre o papel da Psicologia na educação a distância ainda são incipientes, por isso, são recomendados estudos sistemáticos sobre essa temática e a disseminação de ferramentas efetivas nesse campo. Como limitações deste estudo, têmse o número reduzido de bases de dados revisadas e a restrição a publicações em português. Sugere-se, portanto, que estudos futuros revisem outras bases de dados e utilizem referências internacionais com o objetivo de obter uma compreensão mais abrangente das contribuições da Psicologia à educação a distância.

\section{Cristineide Leandro França}

Mestranda no Programa de Pós-Graduação em Psicologia Clínica e Cultura da Universidade de Brasília- UnB, especialista em Psicopedagogia, graduada e licenciada em Psicologia pelo Centro Universitário de João Pessoa, servidora efetiva da Universidade de Brasíla, UNB-Brasília - DF, Brasil.

E-mail: cristineide@unb.br

\section{Karen Weizenmann da Matta}

Mestre em Psicologia Clínica e Cultura pela Universidade de Brasília. Psicóloga graduada na Universidade de Brasília, Brasília - DF, Brasil.

E-mail: karenwm@gmail.com

\section{Elioenai Dornelles Alves}

Doutor em Enfermagem pela Universidade Federal de Santa Catarina. Professor do Departamento de Enfermagem da Universidade de Brasília, Brasília - DF.

E-mail: elioenai@unb.br

\section{Endereço para envio de correspondência:}

QRSW 07 Bloco A-14 Ap. 306 Sudoeste, Brasília - DF. CEP: 70675.714

Recebido 16/1/2010, 1a Reformulação 23/5/2011, Aprovado 17/6/2011. 
Abbad, G., Carvalho, R. \& Zerbini, T. (2006). Evasão em curso via internet: explorando variáveis explicativas. FGV EAESP, 5(2). Recuperado em 10 dezembro, 2009, da RAEeletrônica: www.rae.com.br/eletronica.

Associação Brasileira de Ensino de Psicologia - ABEP. (2007). Grupo de trabalho sobre educação a distância (GT), Brasília. Recuperado em 07 janeiro, 2010, da ABEP:www.abepsi. org.br

Alves, J. R. M. (2009). A história da EAD no Brasil. In Litto F., \& Formiga, M. (Orgs.). Educação a distância: o estado da arte (pp. 39-46). São Paulo: Pearson.

Amaral, R. C., \& Rosini, A. (2009). Concepções de interatividade e tecnologia no processo de tutoria em programas de educação a distância: novos paradigmas na construção do conhecimento. Faculdade Internacional de Curitiba, 4(7), 112. Recuperado em 09 janeiro, 2010, de Intersaberes: http:// intersaberes.grupouninter.com.br/6/2.pdf

Andrade, J. (2007). A mediação na tutoria online: o entrelace que confere significado à aprendizagem. Dissertação de mestrado, Universidade Federal do Ceará e Universidade Norte do Paraná, Salvador, BA.

Aretio, G. L. (1994). Educatión a distancia hoy. Madrid:Uned.

Azambuja, M., \& Guareschi, N. M. F. (2007). Devir vírus. Revista do Departamento de Psicologia - UFF, 19(2), 439-454.
Azevedo, M. A., Guerra, V., Zweiman, B., \& Nishizawa, E. (2001). Educação a distância: o combate à violência doméstica contra crianças e adolescentes - uma experiência bem sucedida. Psicologia e Estudos, 6(1), 85-89.

Bardin, L. (2009). Análise de conteúdo. Edições 70, Brasil

Behar, P., Bercht, M., \& Longhi, M. T. (2007). Integração do humor do aluno no ambiente virtual de aprendizagem ROODA. In Anais do 27 Congresso da SBC, 13 Workshop sobre informática nas escolas. Pelotas, RS.

Bassini, P., Lahud, V. F., \& Lima, C. (2009). Interações educativas em ambientes virtuais: um estudo sobre a constituição de comunidades de aprendizagem. In 19 Simpósio Brasileiro de Informática na Educação - SBIE, Fortaleza, CE.

Bercht, M. (2006). Computação afetiva: vínculos com a psicologia e aplicações na educação. In Prado, O., Fortim, I., \& Consentino, L. (Orgs.). Psicologia \& informática: produções do III. psicoinfo II. Jornada do NPPI (pp. 106-115). São Paulo: Conselho Regional de Psicologia de São Paulo, SP.

Bizzo, N. (2009). Ensino de ciências e EAD. In Litto \& Formiga (Orgs.). Educação a distância: o estado da arte (pp.188-195). São Paulo: Pearson Education do Brasil.

Borba, A., Rangé, B., \& Elia, M. (2007). Disciplina de psicoterapia baseada na WEB: uma estratégia de ensino-aprendizagemavaliação. Anais do XXVII Congresso da SBC, XIII Workshop sobre informática nas escolas, Rio de Janeiro, RJ. 
Carvalho, T., Muller, M., \& Ramos, M. (2005), Ensino a distância: uma proposta de ampliação do estudo em bioética, DST. Jornal Brasileiro de Doenças Sexuais Transmissíveis, 17(3), 211-214.

Carvalho, R., \& Abbad, G. (2006). Avaliação de treinamento a distância: reação, suporte à transferência e impactos no trabalho. Rev. Adm. Contemp., 10(1), 95-116.

Cunha, C., Silva, J., \& Bercht, M. (2008). Proposta de um modelo de atributos para o aprimoramento da comunicação afetiva para professores que atuam na educação a distância. Trabalho apresentado no 19 Simpósio Brasileiro de Informática na Educação, Fortaleza, CE.

Davidoff, L. (2004). Introdução à psicologia (3a ed.). São Paulo: Makron Books.

Lima, E. C. (1990 out./dez.). O conhecimento psicológico e suas relações com a educação. Em Aberto, ano 9(48) 3-20.

Figueiredo, L., K., \& Araújo, R. S. (2009). Educação a distância online e o papel do tutor. Trabalho apresentado no 15 Congresso Internacional ABED de Educação a Distância, Fortaleza, CE.

Filatro, A. (2009). As teorias pedagógicas fundamentais em EAD. In Litto F, \& Formiga, M. (Orgs.). Educação a distância: o estado da arte (pp. 96-104). São Paulo: Pearson.

Fiuza, P. (2002). Aspectos motivacionais na educação a distância: análise estratégica e dimensionamento de ações. Dissertação de mestrado, Universidade Federal de Santa Catarina, Florianópolis, SC.

Formiga, M. (2009). A terminologia da EAD. In Litto, F, \& Formiga, M (Orgs.). Educação a distância: o estado da arte (pp. 39-46). São Paulo: Pearson Education do Brasil.

Gouvêa, F. R., \& Cesar, M. (2009). O professor de EAD: um perfil. Trabalho apresentado no 15 Congresso Internacional ABED de Educação a Distância, Fortaleza, CE.

Guilherme, C., \& Monteiro, A. (2009). Tutoria na formação docente. Trabalho apresentado no 15 Congresso Internacional ABED de Educação a Distância, Fortaleza, CE.

Jaques, P., Lehmann, M., \& Jaques, K. S. (2008). Avaliando a efetividade de um agente pedagógico animado emocional. Trabalho apresentado no Simpósio Brasileiro de Informática na Educação, Fortaleza, CE.

Jaques, P., \& Vicari, R. M. (2005). Estado da arte em ambientes inteligentes de aprendizagem que consideram a afetividade do aluno. Informática na Educação, 8(1), 15-38.

Jensen, L., \& Almeida, O. C. (2009). A correlação entre falta de interatividade e evasão em cursos a distância. Trabalho apresentado no 15 Congresso Internacional ABED de Educação a Distância, Fortaleza, CE.

Laguardia, J., Machado, R., \& Coutinho, E. (2009). Interação nos ambientes virtuais de aprendizagem: análise de dois fóruns de discussão. RECIIS - Revista Eletrônica de Comunicação, Informação \& Inovação em Saúde, 3(2), 37-40.

Lawisncky, F., Haguenauer, C., Cordeiro, F., \& Vinicius, M. (2008). Interação em ambientes virtuais de aprendizagem: análise de uma experiência no curso de pós- graduação em linguística aplicada. Trabalho apresentado no 15
Congresso Internacional ABED de Educação a Distância, Fortaleza, CE.

Litto, F. M. (2009). O atual cenário internacional da EAD. In Litto, F, \& Formiga, M. (Orgs.). Educação a distância: o estado da arte (pp. 14-20). São Paulo: Pearson.

Longhi, M. T., Bercht, M., \& Behar, P., (2008) AnimA: protótipo para reconhecimento dos estados de ânimo no processo de aprendizagem. Trabalho apresentado no II Workshop, Escola de Sistemas de Agentes para Ambientes Colaborativos, EDUNISC, Santa Cruz do Sul, RS.

Longhi, M. T., Pereira, D., \& Behar, P. (2009). Um experimento para compreender como os aspectos afetivos podem ser reconhecidos em ambientes virtuais de aprendizagem. Novas Tecnologias na Educação, 7(1)

Lopes, M. C., \& Xavier S. (2007). A afetividade nas inter-relações professores e alunos no ambiente digital. Revista Brasileira de Aprendizagem Aberta e a Distância, 6, 1-17.

Machado, F., \& Miranda L. (2006). O uso do construtivismo e da afetividade nas metodologias de ensino a distância, PUC, Rio de Janeiro, p. 14, recuperado em 07 janeiro, 2010, de PUC/RJ:www.inf.puc-rio.br/ francis/2006-Construtivismo.pdf

Martins, W., Melo, F., Meireles, V., \& Nalini, L. E. (2003) Sistemas tutores inteligentes em ambiente web baseados em tipos psicológicos. Trabalho apresentado no 10 Congresso Internacional de Educação a Distância, Porto Alegre, RS.

Massimi, M. (1990). História da psicologia brasileira: da época colonial até 1934 (p.70). São Paulo: EPU.

Mattar, J. (2009). Interatividade e aprendizagem. In Litto, F, \& Formiga, M. (Orgs.). Educação a distância: o estado da arte (pp. 112-120). São Paulo: Pearson .

Mehleche, Q., Pereira, A., \& Tarouco, L. (2005). Estratégias de interação entre tutor e estudantes em educação a distância. Novas Tecnologias na Educação, CINTED-UFRGS 3(1), 1-10.

Moore, G. M. (1972). Learner autonomy: The second dimension of independent learning. Convergence, 76-88.

Moran, J. M. (2009). Modelos e avaliação do ensino superior a distância no Brasil. ETD - Educação Temática Digital, 10, (2), 55.

Nunes, I. B. (2009). A história da EAD no mundo. In Litto, F, \& Formiga, M. (Orgs.). Educação a distância: o estado da arte (pp. 2-8). São Paulo: Pearson.

Oliveira, E., Capelo, C., Rego, M., \& Villardi, R. (2004). O processo de aprendizagem em uma perspectiva sócio-interacionista... Ensinar é necessário, avaliar é possível. Trabalho apresentado no 11ํㅡㄹ Congresso Internacional de Educação a Distância, Salvador, BA.

Passarela, L. (2007). O modelo de personalidade de MyersBriggs na educação a distância. Monografia do Curso de Computação, Instituto de Ciências Exatas, Universidade de Brasília, Brasília, DF

Pereira, F., \& Pereira, A. (2003). O psicólogo no Brasil: notas sobre seu processo de profissionalização. Psicologia. Estud. $8,2,19-27$

Pereira, K., Pavanati, I., Maia, L., Fialho, F., Paulista, C., Schruber, J. J. et al. (2007). Uma visão articulada das teorias de Piaget e Vygotsky e suas implicações na educação a distância. Revista Educação em Rede, 2(1). 


\section{Referências}

Perry, W., \& Rumble, G. A. (1987). Short guide to distance education. Cambridge: International Extension College.

Pessotti, I. (1988). Notas para uma história da psicologia brasileira. In Conselho Federal de Psicologia. Quem é o psicólogo brasileiro? (pp.17-31). São Paulo: Edicon.

Peters, O. (1994). Otton Peters on distance education: The industrialization of teaching and learning. New York : Routledge.

Pinto, I. (2009). O problema da evasão nos cursos de EAD. Trabalho apresentado no 15 Congresso Internacional ABED de educação a distância, Fortaleza, CE.

Quarto, C. (2006). Inferindo fatores sócio-afetivos em ambientes de ensino-aprendizagem colaborativos assistidos por computador. Dissertação de mestrado, Universidade Federal do Maranhão, São Luís, MA.

Ramminger, S. (2006). Do encontro ao desencontro: fatores relacionados à procura de cursos de ead em psicologia e à posterior evasão. Dissertação de mestrado, Faculdade de Educação da Pontifícia Universidade Católica do Rio Grande do Sul, Porto Alegre, RS.

Rangé, B. (2001). Programa de treinamento a distância para tratamento do transtorno do pânico e da agorafobia. Rev. Psiq. Clín., 28 (6), 331-339.

Romiszowski, A. J. (2009). Aspectos da pesquisa em EAD. In Litto, F, \& Formiga, M. (Orgs.). Educação a distância: o estado da arte (pp. 422-434). São Paulo: Pearson.

Santana, F., Gaspar, C., Costa, R., Paiva, V., Rodrigues, M. C., \& Alves, E. (2005). Educação a distância nas instituições federais de ensino superior: a situação da enfermagem brasileira. Revista Eletrônica de Enfermagem, 07(1), 41 - 53.

Sá, Z. E. (2007). A dimensão psicossocial da educação a distância. Análise psicodramática. Trabalho apresentado no 13 Congresso Internacional de Educação a Distância, Curitiba, PR.

Santos, M. C. (2009). Afetividade, aprendizagem e avaliação: complementos da tecnologia na Ead virtual. Trabalho apresentado no 15 Congresso Internacional ABED de Educação a Distância, Fortaleza, CE.

Santos, E. M., Neto, J., Araújo, E., Oliveira, S., Barbosa, D., \& Zander, V. (2007). Educação a distância no Brasil: evolução da produção científica. USP/SC - Unicentro. Relatório de pesquisa recuperado em 07 janeiro, 2010, de ABED: http://www.abed. org.br/congresso2007/tc/55200753414PM.pdf

Santos, J. V. (2006). As representações sociais da educação a distância: uma investigação junto a alunos do ensino superior a distância e a alunos do ensino superior presencial. Tese de doutorado, Centro de Filosofia e Ciências Humanas, Universidade Federal de Santa Catarina, Florianópolis, SC.

Saraiva, L., Pernigotti, J., Barcia, R., \& Lapolli, E. (2006). Tensões que afetam os espaços de educação a distância. Psicologia em Estudo, 11(3), 483-491.

Sarmento, D., Barbosa, D. N., \& Mangan, P. (2008). A dimensão didático-pedagógica na educação a distância: um enfoque na prática docente na disciplina de Psicologia da Educação. Novas Tecnologias na Educação, CINTED-UFRGS, 6(1), 1-11.

Sarmet, M., \& Abrahão, J. (2007). O tutor em educação a distância: análise ergonômica das interfaces mediadoras. Educ. Rev., 46, 109-141.
Serra, D. (2005). Afetividade, aprendizagem e educação online. Dissertação de mestrado. Pontifícia Universidade Católica de Minas Gerais, Belo Horizonte, MG.

Silva, J., \& Heck, G. (2008). Utilizando o Questionário MSLQ para identificação de estados afetivos em um ambiente virtual de aprendizagem. Trabalho apresentado no I Seminário de Tecnologia da Informação Aplicada, Chapecó, SC.

Simão, L. M. (2001). Interface gráfica para suporte à percepção emocional em ambientes de cooperação. Dissertação de mestrado, Universidade Federal de Santa Catarina, Florianópolis, SC.

Steil, V. A., Pilon, E., \& Kern, V. (2005). Atitudes com relação à educação a distância em uma universidade. Psicologia em Estudo, 10(2), 253-262.

Tamariz, A., Gonzléz, S., Pessanha, C., \& Braga, E. (2007). Emoções como parte de um ambiente virtual de aprendizagem. Conferência IADIS Ibero-Americana, Universidade Estadual do Norte Fluminense (UENF), Universidade Candido Mendes Campos (UCAM), Rio de Janeiro, RJ.

Tijiboy, A.V., Pereira, E. A., \& Woiciechoski,R.L. (2009). Interação com afeto: aprendizagem em ambientes virtuais de aprendizagem. Novas Tecnologias na Educação, CINTED -UFRGS, $7(1)$

Tijiboy, A. V., Carneiro, M. L., Woiciechoski, L. R., \& Pereira, E. (2009). Compreendendo a mediação do tutor a distância. Novas Tecnologias na Educação, CINTED - UFRGS, 7(1).

Todorov, J. C., Moreira, M., \& Martone, R. (2009). Sistema personalizado de ensino, educação a distância e aprendizagem centrado no aluno. Psicologia: Teoria e Pesquisa, 25(3), 289-296.

Torres, P., \& Fialho, A. (2009). Educação a distância: passado, presente e futuro. Educação a distância: o estado da arte (pp. 456-461). São Paulo: Pearson.

Valentini, C. B., \& Bisol, C. (2008). Análise dos processos cognitivos e autopoiéticos em um ambiente virtual de aprendizagem. Educar, 32, 181-197.

Vedove, J., \& Camargo, R. T. (2009). A influência da empatia na relação tutor-aluno. Faculdade Internacional de Curitiba, 4(7), 1-11. Recuperado em 09 janeiro, 2010, de Intersaberes:http:// intersaberes.grupouninter.com.br/6/3.pdf

Voltolini, R. (2009). Educação a distancia: algumas questões. ETD - Educação Temática Digital, 10(2), 123-139.

Weber, S. (2008). Uma investigação acerca dos fatores que contribuem para o mal-estar e o bem-estar dos professores que trabalham com EAD. Tese de doutorado, Faculdade de Educação, Pontifícia Universidade Católica do Rio Grande do Sul, Porto Alegre, RS.

Zerbini, T. (2007). Avaliação da transferência de treinamento em curso a distância. Tese de doutorado, Instituto de Psicologia, Universidade de Brasília, Brasília, DF.

Zerbini, T., \& Abbad, G. (2009). Reação aos procedimentos instrucionais de um curso via internet: validação de uma escala. Estudos em Psicologia, 26(3), 363-371.

Ziviani, N., Melli, N. C., Neto, J., Araújo, E. M., \& Santos, E. M. (2008). Uma investigação sobre o papel do tutor local e sua visão frente à educação a distância em uma instituição de ensino superior. Trabalho apresentado no 15 Congresso Internacional ABED de Educação a Distância, Fortaleza, CE. 